\title{
Nanofibrous Matrix of Defined Composition Sustains Human Induced Pluripotent Stem Cell Culture
}

\author{
Sara Borrego-González, ${ }^{\dagger}$ Berta de la Cerda, ${ }^{\dagger}$ Francisco J. Díaz-Corrales, and Aránzazu Díaz-Cuenca*
}

Cite This: ACS Appl. Bio Mater. 2021, 4, 3035-3040

Read Online

ABSTRACT: Human induced pluripotent stem cells (hiPSCs) represent the most promising biological material for regenerative medicine applications. In this work, a 3D solid nanofibrous matrix of defined composition (Colamigel-S) consisting of $97 \mathrm{wt} \%$ gelatin, 2.6 wt $\%$ atelocollagen, and $0.4 \mathrm{wt} \%$ laminin has been reproducibly processed and characterized and exhibits a homogeneous nanofibrillar network of high surface area, interconnected microcavities, and typical D-periodic collagen fibril nanostructural features. The purpose of the study was to test the performance of Colamigel-S as substrate for in vitro hiPSCs culture, finding that these cells efficiently attach and grow keeping their characteristic stem morphology and undifferentiated state.

KEYWORDS: biomimetic, microstructure, nanofibrous gelatin, D-periodic collagen, 3D matrix, iPSCs

$\mathrm{T}$ hree-dimensional (3D), nanofibrous structured materials are promising candidates for specific cell culture systems, as they can be utilized to mimic the extracellular environment in vitro, creating an in vivo-like functionality., ${ }^{1,2}$ Multipotent stem cell supportive niches have been identified where anchored cells release signals for self-renewal or lineagespecific differentiation. ${ }^{3}$ The traditional $2 \mathrm{D}$ methods of cell culture lacking the intricacy necessary to mimic the extracellular environment alter shape cell and geometry modifying protein expression and cell fate $e^{4,5}$ and present scalability problems for clinical applications. ${ }^{4}$ Human induced pluripotent stem cells (hiPSCs) can be reprogrammed from adult somatic cells and can be differentiated toward any cell type. hiPSCs are more versatile than multipotent stem cells, are committed to differentiate only to specific lineages, and overcome the ethical concerns associated with the use of embryonic stem cells (ESCs). ${ }^{6}$ Therefore, hiPSCs provide an invaluable contribution for cell-based therapy in regenerative medicine, as well as for disease modeling, pharmaceutical development, and tissue engineering. hiPSCs require a specific culture system for successful maintenance and expansion. At present, despite being expensive and difficult to handle, one of the most widely used growth substrates for feeder-free iPSCs culture is Corning Matrigel Matrix (Matrigel). ${ }^{7}$ This commercial product is not optimal for clinical applications because it is a preparation of solubilized basement membrane from the Engelbreth-Holm-Swarm mouse sarcoma, without a defined chemical composition, presenting the risk of introduction of tumor-derived growth factors and pathogen transmission as well as limiting reproducibility between cultures due to lot-to-lot variability. ${ }^{8}$
In this work, a compositionally defined $3 \mathrm{D}$ nanofibrous matrix, Colamigel-S, is designed to pluripotent stem cell culture purpose and fabricated in the solid form using the temperature induced phase separation (TIPS) technique in combination with a chemical-free cross-linking treatment. Colamigel-S, consists of gelatin as a major matrix component but functionalized with a minimal content of $\mathrm{D}$-structured selfassembled collagen fibrils obtained from atelocollagen, as well as laminin. D-periodicity of collagen fibrils compensates gelatin weakness in relation to cell recognition and cell adhesion properties and, laminin proteins help to maintain pluripotency and drive cell fate specification. ${ }^{8}$ The main innovation and advantages of the presented 3D material to practical application includes: (i) competitive manufacturing cost and simple handling and storage requirements of a $3 \mathrm{D}$ cell culture substrate which, mimics the nanoscale protein fibber meshwork in native ECM; (ii) the main component gelatin, having ECM native collagen amino acid primary structure, represents a safest version of collagen matrices with reduced antigenicity due to heat denaturation; ${ }^{9}$ (iii) the atelocollagen precursor used to synthesize the fibrils of D-periodic functional motifs is a form of collagen without the terminal peptides and has extremely low antigenicity because of the elimination of endogenous antigenic regions and exogenous pathogens by

Received: November 3, 2020

Accepted: April 1, 2021

Published: April 8, 2021 


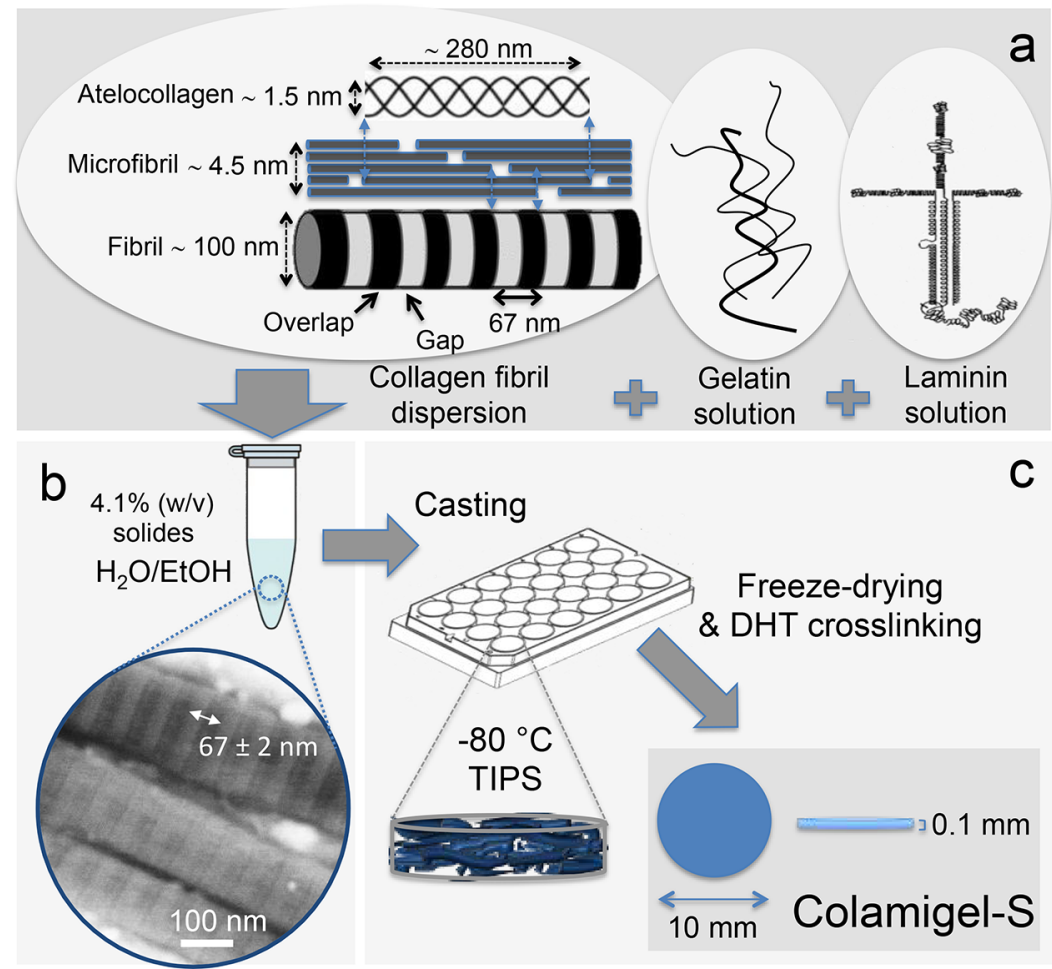

Figure 1. Colamigel-S processing: (a) self-assembled structured collagen fibrils, gelatin and laminin solutions precursor mixture; (b) STEM characterization of $D \approx 67 \mathrm{~nm}$ periodic structure of prepared collagen fibril dispersion; (c) casting, $-80{ }^{\circ} \mathrm{C}$ temperature induced phase separation (TIPS), freeze-drying, and dehydrothermal (DHT) treatment processing route.

proteolytic processing. ${ }^{10}$ As pluripotent state viability of hiPSCs is the initial critical requirement for maintenance, expansion, and therapeutics, the objective of this study was to test the properties of Colamigel-S material as a substrate for hiPSCs in vitro cell culture and its ability to sustain cell viability and stemness compared to a widely used reference material.

$D \approx 67 \mathrm{~nm}$ periodic self-assembled collagen fibril dispersion (Figure $1 \mathrm{~b}$ ), together with gelatin and laminin solutions were processed using the temperature induced phase separation (TIPS) technique. A commercial atelocollagen solution was used as precursor for the preparation of the collagen fibril dispersion. Prepared gelatin/D-periodic collagen/laminin final mixture $(4.1 \%(\mathrm{w} / \mathrm{v})$ total solid content) was cast into the wells of a polystyrene plate and frozen at $-80{ }^{\circ} \mathrm{C}$ to allow the TIPS process. Obtained pieces were freeze-dried and further cross-linked by dehydrothermal (DHT) treatment (Figure 1c). Additionally, matrix pieces of two control materials consisting of single-component, D-periodic structured collagen dispersion $(\mathrm{DCol})$ or bare gelatin $(\mathrm{NfGel}),{ }^{11}$ respectively, were prepared adapting the same procedure. Please see the Supporting Information for the complete and detailed preparation method.

Field-emission gun scanning electron microscopy (FEGSEM) secondary electron images of the final matrix microstructure were obtained on carbon-coated samples. Textural parameters were measured by $\mathrm{N}_{2}$ adsorption-desorption measurements and surface area was calculated from the Brunauer, Emmett, and Teller (BET) method using the adsorption data. XRD and FT-IR analysis of Colamigel-S were compared with the two single-component control materials, gelatin NfGel, and collagen DCol. hiPSCs for cell culture were previously obtained by cell reprogramming from biological material of a healthy donor, after informed consent. ${ }^{12}$ The
hiPSC line used to test Colamigel-S had been previously fully characterized for pluripotency and adapted to grow in feederfree, serum-free conditions (Matrigel substrate and mTeSR1 culture medium). Please see the Supporting Information for more details of materials and methods for the matrices physicochemical characterization, the preparation of culture plates, and the hiPSC culture and assays.

The microstructure of fabricated solid material consisting of $97 \mathrm{wt} \%$ gelatin, $2.6 \mathrm{wt} \%$ atelocollagen, and $0.4 \mathrm{wt} \%$ laminin is shown in Figure 2a, b. FEG-SEM micrographs indicated the formation of a homogeneous nanofibrillar network with fiber diameter within 70-100 $\mathrm{nm}$ and interconnected cavities of 1$2 \mu \mathrm{m}$. Nitrogen physisorption measurements and BET analysis (Figure $2 c, d$ ) indicated a high surface area of $16.5 \pm 0.8 \mathrm{~m}^{2}$ $\mathrm{g}^{-1}$, characteristic of a nanofibrillar material. The diffraction pattern of Colamigel-S is plotted in comparison with the single-component control materials gelatin, NfGel, and nanofibrillar collagen, DCol, (Figure 2e). As expected, bare gelatin NfGel did not show any diffraction peak but the typical amorphous halo from $2 \theta=12^{\circ}$ to $2 \theta=29^{\circ}$. DCol showed characteristic peaks of collagen at $2 \theta=5.5,9.2,21.5$, and $23.9^{\circ}{ }^{13}$ The two firsts peaks at $2 \theta=5.5$ and $9.2^{\circ}$ ( $d$-spacings of 1.6 and $1.0 \mathrm{~nm}$ ) correspond to the intermolecular lateral packing between collagen molecules (equatorial spacing) and the peaks at $2 \theta=21.5$ and $23.9^{\circ}$ ( $d$-spacings of 0.41 and 0.37 $\mathrm{nm}$ ) correspond to the collagen single left-hand helix chain order. $^{14,15}$ XRD pattern of Colamigel-S material confirmed collagen D-periodic fibril nanostructural features, this being particularly relevant as the collagen component is only $2.6 \mathrm{wt}$ $\%$. Although the Colamigel-S infrared analysis presented in Figure $2 \mathrm{f}$ is less conclusive than XRD to assess the long-order material structure, comparison of the gelatin, NfGel, and Colamigel-S infrared bands revealed amide A $\left(\sim 3300 \mathrm{~cm}^{-1}\right.$ 

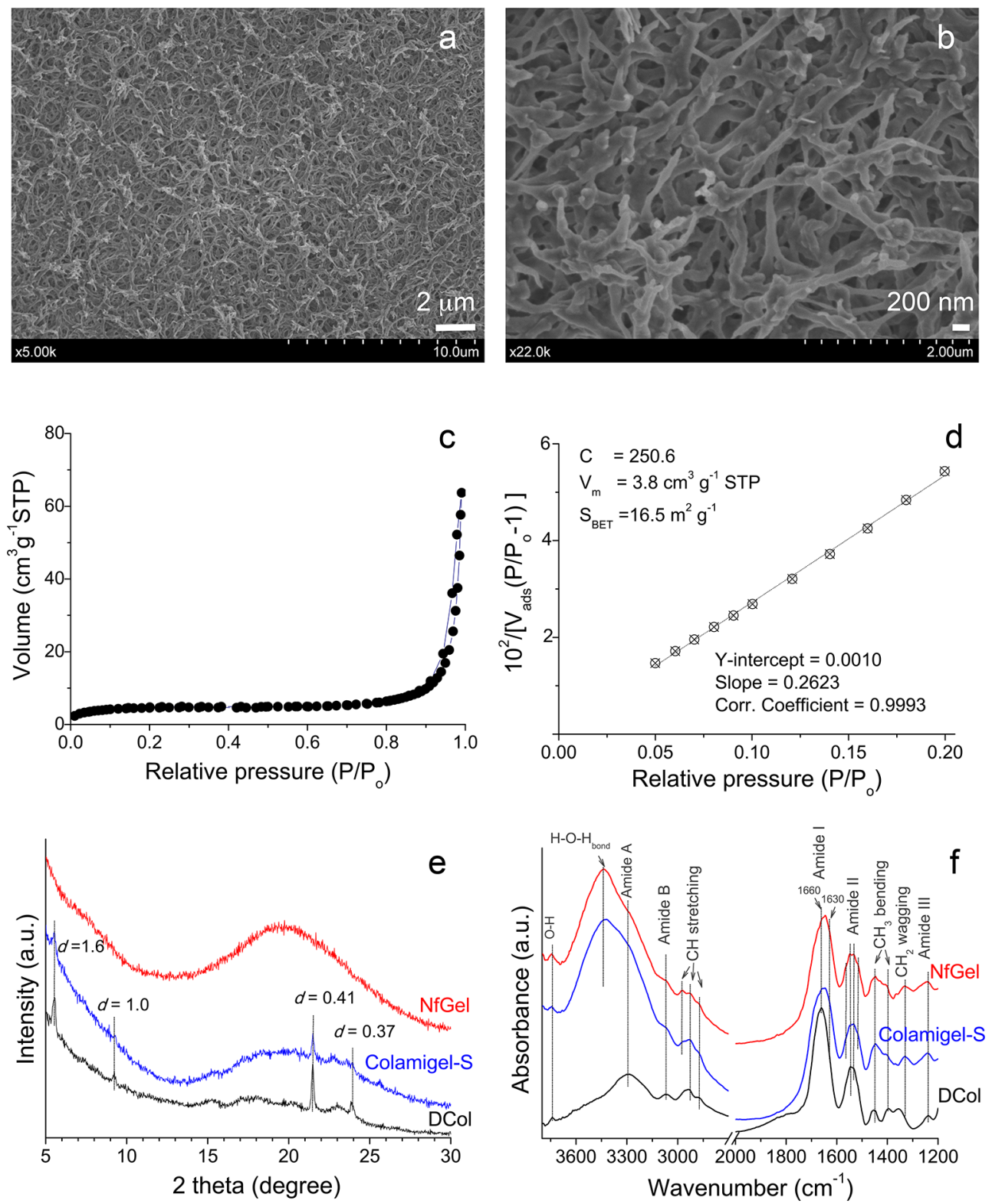

Figure 2. Colamigel-S matrix physicochemical characterization: (a, b) FEG-SEM images; (c, d) $\mathrm{N}_{2}$ adsorption/desorption isotherm and $S_{\mathrm{BET}}$ assessment; (e) XRD ( $d$-spacings in $\mathrm{nm}$ ); and (f) FT-IR analysis of Colamigel-S in comparison with the two control materials prepared using one single component, gelatin NfGel, and collagen DCol.

wavenumbers) and amide I (1660 and $1630 \mathrm{~cm}^{-1}$ intensities ratio) variations, which suggest structured collagen detection for Colamigel-S. ${ }^{16}$

To test the viability of Colamigel-S as a substrate for hiPSC attachment and growth, we seeded the cells on either Matrigel or Colamigel-S or on plates without substrate. hiPSCs proliferate, forming colonies. The growth progression of the hiPSC colonies was followed for 7 days by microscopic examination, finding no cell attachment and no growth in the wells without substrate. Live/dead staining plus Hoechst was used on seeded cells. On both Colamigel-S and Matrigel, similar attachments, viabilities, and growth rates were observed (please see Figure S1 for more results within the studied period of time). After 7 days, hiPSC colonies reached an appropriate size for characterization. hiPSCs grown onto Colamigel-S showed the typical stem morphology of flat colonies with neat borders. The characteristic elevated alkaline phosphatase activity of pluripotent cells was detected as a pink staining (Figure 3, lower panels) and positive live staining for the surface antigen TRA-1-60, distinctive of pluripotent cells, was also shown for the colonies grown onto Colamigel-S (Figure 3, upper panels). No difference could be appreciated with the reference material Matrigel.

Colamigel-S, based on gelatin as the major component, incorporates a minority amount of D-periodic atelocollagen and laminin, which have been shown as critical elements to allow hiPSC adhesion and growth to the material. This effect was confirmed after the observation of viability failure when using the control group corresponding to iPSCs seeded on similarly processed nanofibrous single-component gelatin.

For additional characterization of the hiPSCs grown onto Colamigel-S substrate, we tested the protein expression and subcellular localization of characteristic markers of stemness, specifically, the transcription factors OCT4 and NANOG and 


\section{Colamigel-S}
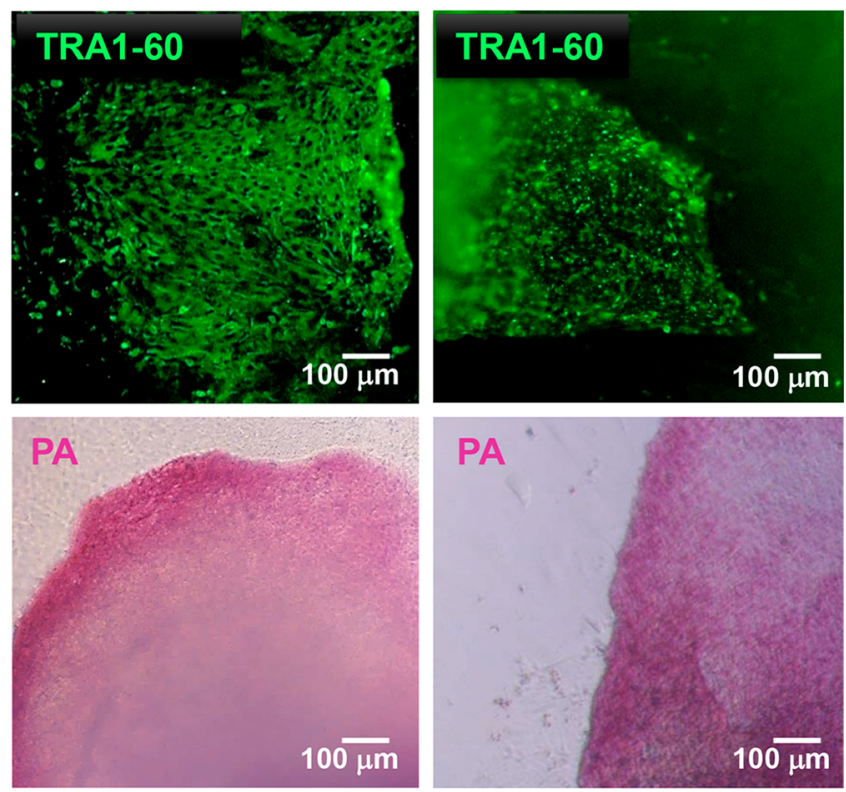

Figure 3. In situ characterization of the colony morphology and pluripotent state of hiPSCs grown on Colamigel-S and Matrigel. Top row, live-staining for TRA-1-60, in green. Lower row, alkaline phosphatase activity, in pink. the cell surface antigens SSEA4 and TRA-1-81. Technically, double immunostainings were performed. As a result, every row in Figure 4 shows, from left to right, images of one hiPSC colony stained for nuclei (DAPI), for a pluripotency marker located at the cell surface, for a pluripotency marker of nuclear localization, and finally, a combined image. In the DAPI and MERGE images of Figure 4, hiPSC colonies showed the characteristic stem morphology of flat colonies formed by a compact mass of cells with high nuclear/cytoplasmic ratio. Positive labeling for TRA-1-81, NANOG, SSEA4, and OCT4, including their expected subcellular localization, demonstrated that Colamigel-S substrate provided the conditions required for the undifferentiated growth of hiPSCs and the maintenance of their specific features with no difference compared to the control substrate Matrigel (see Figures S2 for more results within the studied period of time).

Importantly, the study demonstrated the suitability of 3D nanofibrous structures for sustained hiPSCs plutipotency and ratifies other recent reports using fibrous materials obtained by electrospinning of synthetic polymers. Culture of hiPSCs on PMEDSAH:PLGA nanofibers has pointed out that the 3D architecture of the membranes itself was critical for maintaining the cell pluripotency state, ${ }^{17}$ whereas fibrous microscale polystyrene scaffolds have been suggested to efficiently trap hiPSCs, leading to a large number of pluripotent colonies. ${ }^{18}$ Also related, 3D collagen scaffolds have been shown to increase mouse embryonic fibroblast
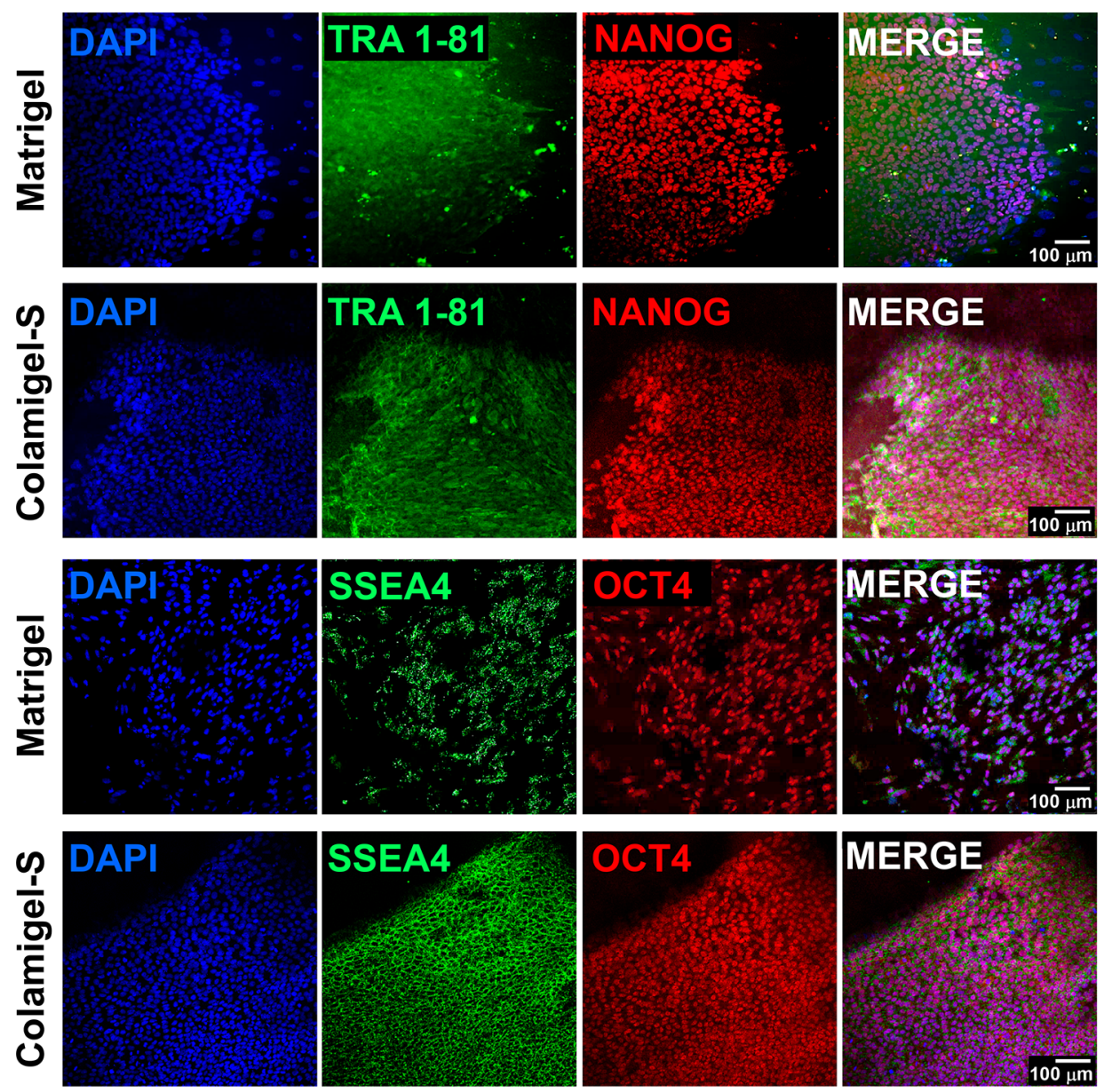

Figure 4. Characterization of hiPSCs grown onto Colamigel-S or Matrigel by immunofluorescence staining for a panel of stem markers, with DNA stained in blue, cell surface markers in green, and nuclear markers in red. 
reprogramming efficiency, promoting iPSCs induction with higher pluripotency in comparison with the conventional $2 \mathrm{D}$ method. ${ }^{19}$

This work formulation then provides not only a biomimetic composition but also a network of nanofibers within 70-100 $\mathrm{nm}$ diameters, more similar to the natural ECM dimensions than achieved for electrospun materials, which reported thicker fiber diameters closer to $1 \mu \mathrm{m}$, and above. In addition, the proposed chemically defined synthesis of Colamigel-S, based on highly purified atelocollagen monomers and gelatin, eliminates some of the important drawbacks in the production of collagen biomaterials based on animal extracted polymeric collagen such as the batch-to-batch variability and the risk of including impurities or infectious agents. ${ }^{10,20}$ Colamigel-S processing, using the TIPS technique and a chemical-free cross-linking method, produces a nanofibrous structured material without the use of toxic solvents or reagents. This represents an important advantage of Colamigel-S in comparison with the materials obtained by the electrospining technique. Hence, Colamigel-S, processed in solid form, could well serve as a transfer vehicle of iPSCs to allow straightforward surgical implantation.

The Colamigel-S substrate can be tested in the future for other cell culture and differentiation purposes once it has demonstrated its compatibility with hiPSCs and its ability as a growing substrate for the maintenance and amplification of these cells, with the added value, compared to the standard substrate Matrigel, of defined composition, easy handling and affordable cost of its components.

In conclusion, the toxin-free processed $3 \mathrm{D}$ nanofibrous matrix of defined composition and solid form, Colamigel-S, obtained using natural precursors formulation of reduced antigenicity, promotes the attachment of human iPSCs under feeder-free, serum-free culture conditions and efficiently sustains their growth, keeping their characteristic morphology and preserving their undifferentiated state.

\section{ASSOCIATED CONTENT}

\section{SI Supporting Information}

The Supporting Information is available free of charge at https://pubs.acs.org/doi/10.1021/acsabm.0c00425.

Materials and methods: detailed protocol of the preparation of the nanofibrous matrix (Colamigel-S), Colamigel-S material physicochemical characterization, preparation of cell culture plates and human iPSC culture and assays; additional live/dead plus Hoechts assay images and hiPSC characterization for days two and four (PDF)

\section{AUTHOR INFORMATION}

\section{Corresponding Author}

Aránzazu Díaz-Cuenca - Materials Science Institute of Seville (ICMS), Joint CSIC-University of Seville Center, Seville 41092, Spain; Networking Research Center on Bioengineering, Biomaterials and Nanomedicine (CIBER$B B N)$, Madrid 28029, Spain; 이잉.org/0000-00025790-4452; Email: aranzazu@icmse.csic.es

\section{Authors}

Sara Borrego-González - Materials Science Institute of Seville (ICMS), Joint CSIC-University of Seville Center, Seville 41092, Spain; Networking Research Center on
Bioengineering, Biomaterials and Nanomedicine (CIBERBBN), Madrid 28029, Spain

Berta de la Cerda - Department of Cell Therapy and Regeneration, Andalusian Molecular Biology and Regenerative Medicine Centre (CABIMER), Seville 41092, Spain

Francisco J. Díaz-Corrales - Department of Cell Therapy and Regeneration, Andalusian Molecular Biology and Regenerative Medicine Centre (CABIMER), Seville 41092, Spain

Complete contact information is available at:

https://pubs.acs.org/10.1021/acsabm.0c00425

\section{Author Contributions}

${ }^{\dagger}$ S.B. and B.C. contributed equally to this work.

\section{Notes}

The authors declare no competing financial interest.

\section{ACKNOWLEDGMENTS}

The authors gratefully acknowledge the financial support provided by the Andalusian Ministry of Economy, Science and Innovation (Proyecto Excelencia P10-CTS-6681) and Andalusian Ministry of Health (PI-0099-2018).

\section{REFERENCES}

(1) Radvar, E.; Azevedo, H. S. Supramolecular Nanofibrous Peptide/ Polymer Hydrogels for the Multiplexing of Bioactive Signals. ACS Biomater. Sci. Eng. 2019, 5, 4646-4656.

(2) Frantz, C.; Stewart, K. M.; Weaver, V. M. The extracellular matrix at a glance. J. Cell Sci. 2010, 123, 4195-4200.

(3) Gattazzo, F.; Urciuolo, A.; Bonaldo, P. Extracellular matrix: a dynamic microenvironment for stem cell niche. Biochim. Biophys. Acta, Gen. Subj. 2014, 1840, 2506-2519.

(4) McKee, C.; Chaudhry, G. R. Advances and challenges in stem cell culture. Colloids Surf., B 2017, 159, 62-77.

(5) Thomas, C. H.; Collier, J. H.; Sfeir, C. S.; Healy, K. E. Engineering gene expression and protein synthesis by modulation of nuclear shape. Proc. Natl. Acad. Sci. U. S. A. 2002, 99, 1972-1977.

(6) Blau, H. M.; Daley, G. Q. Stem Cells in the Treatment of Disease. N. Engl. J. Med. 2019, 380, 1748-60.

(7) Chen, K. G.; Mallon, B. S.; McKay, R. D. G.; Robey, P. G. Human pluripotent stem cell culture: considerations for maintenance, expansion, and therapeutics. Cell Stem Cell 2014, 14, 13-26.

(8) Hagbard, L.; Cameron, K.; August, P.; Penton, C.; Parmar, M.; Hay, D. C.; Kallur, T. Developing defined substrates for stem cell culture and differentiation. Philos. Trans. R. Soc., B 2018, 373, 20170230.

(9) Santoro, M.; Tatara, A. M.; Mikos, A. G. Gelatin carriers for drug and cell delivery in tissue engineering. J. Controlled Release 2014, 190, 210-218.

(10) Ogawa, S.; Onodera, J.; Honda, R.; Fujimoto, I. Influence of systemic administration of atelocollagen on mouse livers: an ideal biomaterial for systemic drug delivery. J. Toxicol. Sci. 2011, 36, 751762 .

(11) Borrego-González, S.; Romero-Sánchez, L. B.; Blázquez, J.; Díaz-Cuenca, A. Nanostructured hybrid device mimicking bone extracellular matrix as local and sustained antibiotic delivery system. Microporous Mesoporous Mater. 2018, 256, 165-176.

(12) García Delgado, A. B.; de la Cerda, B.; Alba Amador, J.; Valdés Sanchez, M. L.; Fernández-Muñoz, B.; Relimpio López, I.; Rodríguez de la Rua, E.; Díez Lloret, A.; Calado, S. M.; Sánchez Pernaute, R.; Bhattacharya, S. S.; Díaz Corrales, F. J. Subretinal Transplant of Induced Pluripotent Stem Cell-Derived Retinal Pigment Epithelium on Nanostructured Fibrin-Agarose. Tissue Eng., Part A 2019, 25, 799808. 
(13) Thanikaivelan, P.; Narayanan, N. T.; Pradhan, B. K.; Ajayan, P. $\mathrm{M}$. Collagen based magnetic nanocomposites for oil removal applications. Sci. Rep. 2012, 2, 230.

(14) Fratzl, P.; Weinkamer, R. Nature's hierarchical materials. Prog. Mater. Sci. 2007, 52, 1263-1334.

(15) Mehta, A.; Raghava Rao, J.; Fathima, N. N. Electrostatic Forces Mediated by Choline Dihydrogen Phosphate Stabilize Collagen. J. Phys. Chem. B 2015, 119, 12816-12827.

(16) Romero-Sánchez, L. B.; Borrego-González, S.; Díaz-Cuenca, A. High surface area biopolymeric-ceramic scaffolds for hard tissue engineering. Biomed. Phys. Eng. Express 2017, 3, 035012.

(17) Alamein, M. A.; Wolvetang, E. J.; Ovchinnikov, D. A.; Stephens, S.; Sanders, K.; Warnke, P. H. J. Tissue Eng. Regener. Med. 2015, 9, 1078-1083.

(18) Leong, M. F.; Lu, H. F.; Lim, T. C.; Du, C.; Ma, N. K. L.; Wan, A. C. A. Acta Biomater. 2016, 46, 266-277.

(19) Gu, Q.; Zhu, H.; Chen, L.; Shuai, L.; Fang, J.; Wu, J.; Liu, L.; Li, W.; Dai, J.; Hao, J.; Zhou, Q. Protein Cell 2016, 7 (11), 844-848. (20) Lynn, A. K.; Yannas, I. V.; Bonfield, W. Antigenicity and immunogenicity of collagen. J. Biomed. Mater. Res. 2004, 71B, 343354. 